\title{
Chemical abundances of 10 metal-poor halo stars
}

\author{
G. S. Bai ${ }^{1}$, G. Zhao ${ }^{1}$, Y. Q. Chen ${ }^{1}$, J. R. Shi ${ }^{1}$, V. G. Klochkova ${ }^{2}$, V. E. Panchuk ${ }^{2}$, H. M. Qiu ${ }^{1}$, and H. W. Zhang ${ }^{1,3}$ \\ 1 National Astronomical Observatories, Chinese Academy of Sciences, Beijing 100012, PR China \\ e-mail: bgs@bao.ac.cn \\ 2 Special Astrophysical Observatory, Russian Academy of Sciences, Karachai-Cirkassian Republic, \\ Nizhnij Arkhyz 369167, Russia \\ 3 Department of Astronomy, School of Physics, Peking University, Beijing 100871, PR China
}

Received 27 November 2003 / Accepted 3 May 2004

\begin{abstract}
We present observations of ten metal-poor halo stars with the metallicity range $-2.3<[\mathrm{Fe} / \mathrm{H}]<-1.4$ and derive their stellar parameters, acquire some elemental abundances relative to iron and discuss the relation between the abundance ratio and the metallicity. It was found that oxygen abundances are nearly constant at a level of 0.6 dex for our metal-poor halo stars when the non-LTE correction is considered. The $\alpha$-elements $(\mathrm{Mg}, \mathrm{Si}, \mathrm{Ca}$ and $\mathrm{Ti}$ ) are overabundant relative to $\mathrm{Fe}$ and decrease with increasing metallicity. We also obtained a significant underabundant non-LTE $[\mathrm{Na} / \mathrm{Fe}]$ ratio from $\mathrm{Na}$ I $\mathrm{D}$ lines which have a large deviation from the LTE assumption. Scandium is marginally overabundant with respect to iron and tends to decrease with increasing metallicity like the $\alpha$-elements. A nearly solar value of $[\mathrm{Cr} / \mathrm{Fe}]$ ratio and underabundant $[\mathrm{Mn} / \mathrm{Fe}] \mathrm{ratio}$ are obtained.
\end{abstract}

Key words. stars: abundances - stars: fundamental parameters - Galaxy: abundances - Galaxy: halo

\section{Introduction}

Abundance analysis of the stellar atmosphere for different population stars provides a constraint for the model of Galactic evolution and nucleosynthesis. Specifically, the investigations of chemical composition of low mass metal-poor stars $(M<$ $\left.0.9 M_{\odot},[\mathrm{Fe} / \mathrm{H}]<-1.0\right)$ which have retained the initial material they formed in the early Galaxy is important means of the understanding an original Galactic formation. The overabundances of $\alpha$-elements relative to iron observed in old stars (Zhao \& Magain 1990; Gratton et al. 1997, 2003) has been seen, but different trends are obtained by other authors (Nissen et al. 1994; Stephens \& Boesgaard 2002). Determination of oxygen abundance is one of the most controversial and interesting topics, especially the behavior of the $[\mathrm{O} / \mathrm{Fe}]$ ratio in metal-poor halo stars. There are several indicators of oxygen abundance for metal-poor stars, but the different indicators give different results: plateau-like $[\mathrm{O} / \mathrm{Fe}]$ from $[\mathrm{O} \mathrm{I}]$ lines, and rising $[\mathrm{O} / \mathrm{Fe}]$ from $\mathrm{O}$ I triplets or $\mathrm{OH}$ lines (see, e.g., Boesgaard et al. 1999; Mishenina et al. 2000; Allende Prieto et al. 2001; Nissen et al. 2002; Takeda 2003). [O I] lines are considered more reliable indicators due to their weak $T_{\text {eff }}$ sensitivety as well as the general belief of the validity for the assumption of LTE, while $\mathrm{O}$ I and $\mathrm{OH}$ lines are less reliable because of the uncertainties involved in the non-LTE correction ( $\mathrm{O}_{\text {I triplets) }}$ or with the effect of atmospheric inhomogeneity $(\mathrm{OH}$ lines, Asplund \& García Pérez 2001; Takeda 2003). In this paper, we discuss $[\mathrm{O} / \mathrm{Fe}]$ abundance using $\mathrm{O}_{\mathrm{I}}$ triplets with the consideration of non-LTE corrections. The heavy elements in the Universe have been synthesized by a variety of nucleosynthesis processes. Fe-group elements are mainly produced in supernovae with less massive progenitors (type Ia supernovae), although a non-negligible amount can also be produced in type II supernovae (Nomoto et al. 1984). All elements heavier than the Fe-group are synthesized predominantly by neutron capture reactions that have often referred to as s-process or r-process elements (Wheeler et al. 1989). In recent years, the heavy element abundance of very metal poor stars has been of special interest, as it offers an opportunity to study the astrophysical neutron-capture processes (e.g., Ryan et al. 1996; Norris et al. 2001).

Although the abundances of halo stars have been studied in many extensive papers (e.g., McWilliam et al. 1995; Gilmore \& Wyse 1998) they are still of high interest in studying the accretion history of our Galaxy with companion galaxies. Nissen $\&$ Schuster (1997) found a group of the reduced $[\alpha / \mathrm{Fe}]$ halo stars which belong to the outer halo with the very large $R_{\max }$ (the maximum distance from the Galactic center) and $\mathcal{Z}_{\max }$ (the maximum distance from the Galactic plane). Combined with the $[\mathrm{Na} / \mathrm{Fe}]$ and $[\mathrm{Ni} / \mathrm{Fe}]$ results, they suggested that $\alpha$-poor halo stars have been accreted from dwarf galaxies with a different chemical evolution history from the Milky Way. Hanson et al. (1998) found that a total of $20 \%$ of retrograde giant sample stars and $13 \%$ of all halo giant sample stars have low $[\mathrm{Na} / \mathrm{Fe}]$ ratios, and suggest that there may be a group of stars significantly younger than the typical halo giants. In 
Table 1. The basic parameters and observational data of sample stars.

\begin{tabular}{rlrrcccccc}
\hline \hline No. & Star & $V$ & $b-y$ & $m_{1}$ & $c_{1}$ & $\pi(\mathrm{mas})$ & $\sigma(\pi)$ & Obs. date & $S / N$ \\
\hline 1 & HD 108177 & 9.66 & 0.331 & 0.060 & 0.285 & 10.95 & 1.29 & $2001 / 04 / 25$ & 260 \\
2 & HD 149414 & 9.60 & 0.474 & 0.202 & 0.159 & 20.71 & 1.50 & $2001 / 04 / 17$ & 218 \\
3 & $\mathrm{BD}+02^{\circ} 3375$ & 9.94 & 0.352 & 0.047 & 0.258 & 8.35 & 1.64 & $2001 / 04 / 17$ & 263 \\
4 & $\mathrm{BD}+20^{\circ} 3603$ & 9.77 & 0.323 & 0.048 & 0.308 & 6.47 & 7.85 & $2001 / 04 / 16$ & 218 \\
5 & $\mathrm{BD}+26^{\circ} 2606$ & 9.73 & 0.332 & 0.052 & 0.289 & 10.28 & 1.42 & $2001 / 04 / 17$ & 240 \\
6 & $\mathrm{BD}+28^{\circ} 2137$ & 10.89 & 0.322 & 0.055 & 0.321 & 5.35 & 1.94 & $2001 / 04 / 23$ & 240 \\
7 & $\mathrm{BD}+29^{\circ} 2091$ & 10.26 & 0.382 & 0.059 & 0.157 & 10.55 & 1.75 & $2001 / 04 / 18$ & 170 \\
8 & $\mathrm{BD}+34^{\circ} 2476$ & 10.05 & 0.309 & 0.055 & 0.354 & 5.37 & 1.60 & $2001 / 04 / 17$ & 260 \\
9 & $\mathrm{BD}+42^{\circ} 2667$ & 9.86 & 0.342 & 0.067 & 0.277 & 8.05 & 1.12 & $2002 / 04 / 24$ & 207 \\
10 & $\mathrm{BD}+51^{\circ} 1696$ & 9.91 & 0.398 & 0.099 & 0.179 & 13.61 & 1.54 & $2001 / 04 / 21$ & 170 \\
\hline
\end{tabular}

particular, recent abundances of giant stars in dwarf galaxies (Shetrone et al. 2003) show that these stars have underabundant $[\mathrm{Na} / \mathrm{Fe}]$ and $[\mathrm{Ni} / \mathrm{Fe}]$ and fit the $[\mathrm{Na} / \mathrm{Fe}]-[\mathrm{Ni} / \mathrm{Fe}]$ relation found in Nissen \& Schuster (1997). We thus investigate the abundance nature for 10 halo stars, most of which are on retrograde orbits.

In this paper we analyze the chemical composition of 10 halo stars based on the medium-resolution and high signal-to-noise ratio spectra obtained with the $6 \mathrm{~m}$ telescope of the Special Astrophysical Observatory in Russia. These sample stars with high proper motion and the low mass $\left(M<0.9 M_{\odot}\right)$ contain information of chemical enrichment history of the Milky Way. The abundance analysis of $\alpha$-elements $(\mathrm{O}, \mathrm{Mg}, \mathrm{Si}$, $\mathrm{Ca}, \mathrm{Ti}), \mathrm{Na}, \mathrm{Sc}$, iron-peak elements ( $\mathrm{Cr}$ and $\mathrm{Mn})$ and the neutron capture element $\mathrm{Ba}$ are given. Among these elements, we focused on a few elements, such as $\mathrm{O}, \mathrm{Na}, \mathrm{Sc}$ and $\mathrm{Mn}$, which are not well studied or have no definitive results in metal-poor stars.

\section{Observations and data reduction}

We selected a sample of 10 metal-poor stars with the metallicity range $-2.3<[\mathrm{Fe} / \mathrm{H}]<-1.4$ from Schuster \& Nissen (1989). All of these stars have high proper motions and slow rotatings $V_{\text {rot }}<100 \mathrm{~km} \mathrm{~s}^{-1}$ that combine both kinematics and metallicity information of halo component (Nissen \& Schuster 1991). The sample stars were observed using the $6 \mathrm{~m}$ telescope of the Special Astrophysical Observatory with the ECHELLE spectrometer PFES equipped with the CCD $\left(1040 \times 1160\right.$ pixels, the pixel size $\left.16 \times 16 \mu \mathrm{m}^{2}\right)$. The spectral wavelength coverage is of 4300-7980 $\AA$ with the resolving power of 15000 and the signal-to-noise ratio is about 200. The basic parameters and observational data are summarized in Table 1. The Cols. 3-8 list the visual magnitude, colour indices and Hipparcos parallaxes of sample stars from SIMBAD database. The final two columns give the observational date and the signal-to-noise ratio.

The data reduction is processed with ECHELLE package of standard ESO MIDAS routines for order identification, background subtraction, flat-field correction, order extraction and wavelength calibration. Then the spectrum was normalized by a continuum function determined by fitting a spline curve to a set of pre-selected continuum windows which were estimated from the solar atlas after the correction for the radial velocity shift measured from at least 20 lines. Finally, the equivalent widths $(E W)$ were measured by two methods: direct integration and Gaussian profile fitting.

The accuracy of the equivalent widths is estimated by comparing them with the other measurements for stars in common. Zhao \& Magain (1990) observed BD $+02^{\circ} 3375$ at the European Southern Observatory $3.6 \mathrm{~m}$ telescope using CASPEC $(R \sim$ $20000, S / N \geq 100$ ). Stephens \& Boesgaard (2002) observed $\mathrm{BD}+42^{\circ} 2667$ using the KPNO Mayall $4 \mathrm{~m}$ telescope with CASPEC $(R \sim 35000, S / N \sim 240)$. Figure 1 shows the comparisons of these two sets of data. The systematic difference between the two sets of data are small and linear least squares fittings give:

$$
\begin{aligned}
E W(\mathrm{ZM} 90)= & 0.968( \pm 0.016) E W(\text { this work }) \\
& +1.463( \pm 0.542), \text { for } \mathrm{BD}+02^{\circ} 3375 . \\
E W(\mathrm{SB} 02)= & 0.973( \pm 0.018) E W(\text { this work }) \\
& +0.054( \pm 0.723), \text { for } \mathrm{BD}+42^{\circ} 2667 .
\end{aligned}
$$

The standard deviations of the two relations are $3.04 \mathrm{~m} \AA$ (for 63 lines) and $3.52 \mathrm{~m} \AA$ (for 98 lines). The scatter in Zhao \& Magain (1990) measurements was $3 \mathrm{~m} \AA$ which is quoted as an upper limit on the random errors. Stephens \& Boesgaard (2002) gave the residuals less than $2.5 \mathrm{~m} \AA$ of scatter. Therefore we estimate that the typical uncertainty of the $E W$ measurements in this study is about $3 \mathrm{m \AA}$.

\section{Stellar atmospheric parameters and kinematics}

For the establishment of the atmospheric model, we need to derive the values of four stellar atmospheric parameters: effective temperature, surface gravity, metallicity and microturbulence. The effective temperature was determined from the Strömgren photometric indices $(b-y), c_{1}$ and metallicity $[\mathrm{Fe} / \mathrm{H}]$ using the calibration of Alonso et al. (1996). The typical error of effective temperature is estimated to be about $100 \mathrm{~K}$. The gravities are determined from the abundance analysis by requiring that FeI and FeII lines give the same iron abundance. Another group 

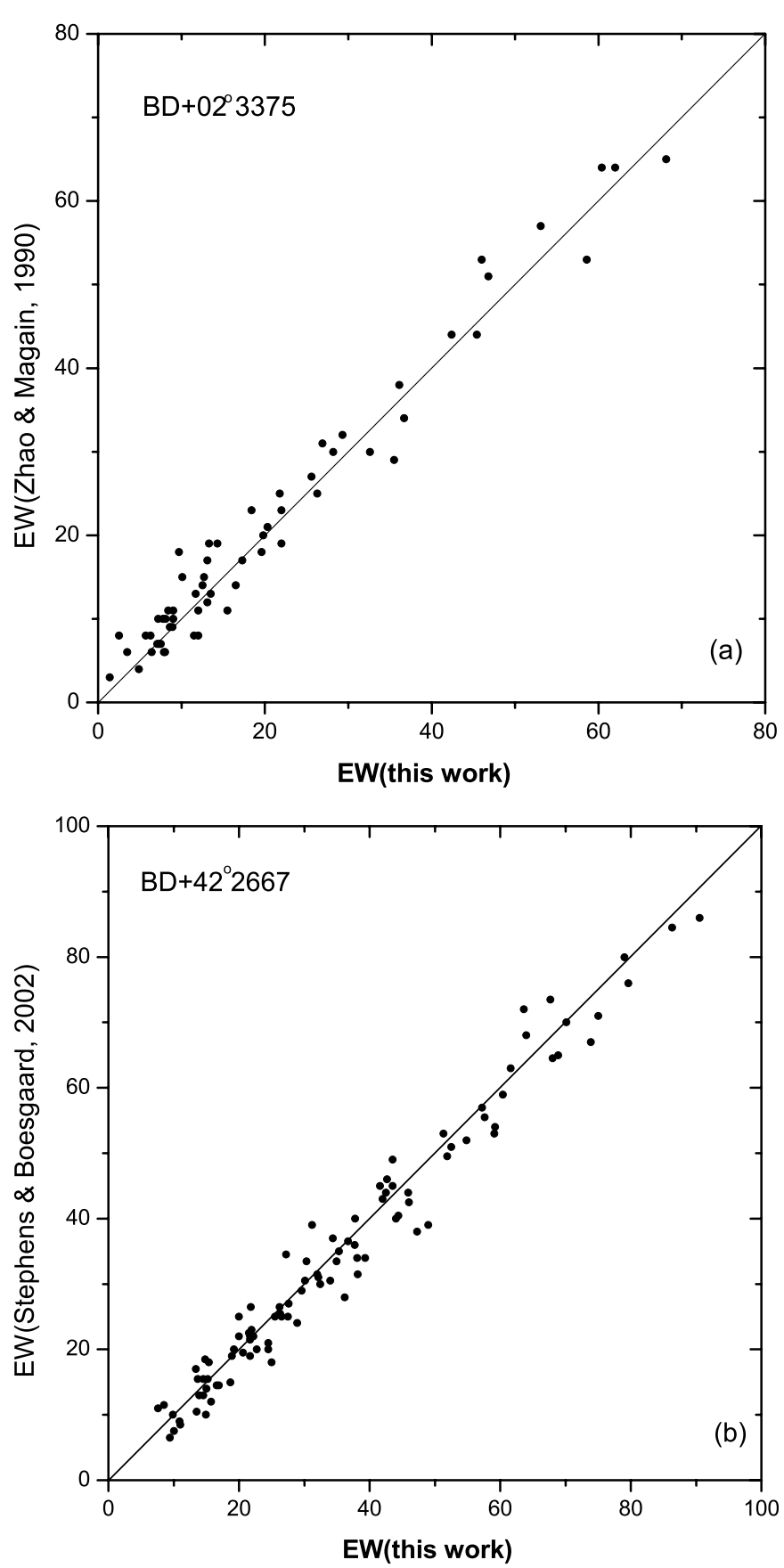

Fig. 1. The comparison of equivalent widths with other works: a) with Zhao \& Magain (1990) for $\mathrm{BD}+02^{\circ} 3375$, b) with Stephens \& Boesgaard (2002) for BD $+42^{\circ} 2667$.

of gravities calculated using Hipparcos parallaxes following Nissen et al. (1997) are nearly similar to these. Because the several program stars have large standard errors of Hipparcos parallaxes $\sigma(\pi)$, we adopt the first group of gravities (Col. 3 of Table 2) and estimate the uncertainties as 0.3 dex. The metallicity was first derived from the Strömgren $\mathrm{m}_{1}$ index using the calibrations of Schuster \& Nissen (1989), but the final metallicity was determined from the abundance analysis of $\mathrm{Fe}$ lines. The whole procedure of determining $T_{\text {eff }}, \log g$ and metallicity was repeated until consistency was reached. Finally, the initial microturbulence velocities for all stars were $1.5 \mathrm{~km} \mathrm{~s}^{-1}$ then adjusted from the abundance analysis by requiring a zero slope of $[\mathrm{Fe} / \mathrm{H}]$ vs. $E W$. Uncertainties in the adopted microturbulences are likely to be about $0.3 \mathrm{~km} \mathrm{~s}^{-1}$. Stellar parameters of sample stars are presented in Table 2.

As a check of the parameters used here, we compare our values with those determined by other studies. Alonso et al. (1996) used the InfraRed Flux Method (IRFM, Blacwell et al. 1990) to determine the mean $T_{\text {eff }}$ values from $J, H$ and $K$ band for a large number of stars. Nissen et al. (1997) also determined $T_{\text {eff }}$ values for a large number of stars using photometric indices $(b-y)$. Fulbright (2000) adjusted $T_{\text {eff }}$ so that the Fe I lines with high excitation potential $(E P)$ give the same iron abundance as those with lower EPs. Tomkin et al. (1992) adopted $T_{\text {eff }}$ values based on the red and near-infrared colors and the temperature-color calibration of Peterson \& Carney (1979), and their data are used by Takeda (2003) to calculate non-LTE oxygen abundances. Figure 2 shows the $T_{\text {eff }}$ comparison of this work with these studies. Our $T_{\text {eff }}$ values agree well with the results of Nissen et al. (1997) and almost accord with Alonso et al. (1996) except for two stars, which have deviations larger than the standard deviation of calibration. In Fulbright (2000), there are two common stars having a large deviation than us. They determined $T_{\text {eff }}$ from the excitation balance of Fe I lines. Takeda (2003) used systematically lower $T_{\text {eff }}$ values than ours in the high temperature range.

The Cols. 8 to 10 of Table 2 list space velocities $U, V$ and $W$ with respect to the Local Standard of Rest (LSR), that are calculated based on the method of Johnson \& Soderblom (1987), and the total space velocities $v_{\text {tot }}=\left(U^{2}+V^{2}+W^{2}\right)^{1 / 2}$ in Col. 11 . In the Toomre diagram Fig. 3 the location of sample stars are agreement with the criterion of Fuhrmann (2002) for halo stars, i.e. $v_{\text {tot }}>180 \mathrm{~km} \mathrm{~s}^{-1}$. Moreover, most stars have $V_{\mathrm{LSR}}<$ $-220 \mathrm{~km} \mathrm{~s}^{-1}$; they are related on retrograde orbits, except for one star, $\mathrm{BD}+26^{\circ} 2606$, which is a halo star with a thick-disk orbit. Particularly, five stars show $V_{\mathrm{LSR}}<-260 \mathrm{~km} \mathrm{~s}^{-1}$ and are located outside the $v_{\text {tot }}=300 \mathrm{~km} \mathrm{~s}^{-1}$ circle in the Toomre diagram and they have definitively retrograde orbits which are shown by filled circles in this figure and the following figures.

\section{Model atmospheres and abundance determinations}

The abundance analysis is based on flux constant, homogeneous and LTE model atmospheres taken from Kurucz (1993). The elemental abundances were derived by requiring that the calculated equivalent widths from the models should match the observed ones. Some accurate atomic line data such as oscillator strengths $(\log g f)$ and enhancement factors $\left(f_{6}\right)$ are required. The oscillator strengths used in this study are taken from the NIST database (http://www.nist.gov), and the enhancement factors are taken from the literatures (Chen et al. 2000, and see references therein). All atomic line data used in the present work and the $E W$ values of program stars are available in electronic form at the CDS via anonymous ftp to cdsarc.u-strasbg.fr (130.79.128.5) or via http://cdsweb.u-strasbg.fr/cgi-bin/qcat?J/A+A/425/671

The abundance errors are mainly from two parts: the systematic errors include the uncertainty of stellar parameters and 
Table 2. The stellar parameters and kinematics.

\begin{tabular}{lccccccrrrr}
\hline \hline Star & $T_{\text {eff }}$ & $\log g$ & $\log g$ (par.) & {$[\mathrm{Fe} / \mathrm{H}]$} & $\xi_{t}$ & Mass $\left(M_{\odot}\right)$ & $U_{\text {LSR }}$ & $V_{\text {LSR }}$ & $W_{\text {LSR }}$ & $v_{\text {tot }}$ \\
\hline HD 108177 & 6036 & 4.35 & 4.34 & -1.69 & 1.50 & 0.70 & -118 & -224 & 59 & 260 \\
HD 149414 & 5090 & 4.60 & 4.45 & -1.51 & 1.50 & $<0.70$ & 78 & -167 & -130 & 226 \\
$\mathrm{BD}+02^{\circ} 3375$ & 5926 & 4.20 & 4.14 & -2.25 & 1.50 & 0.70 & 356 & -246 & 85 & 441 \\
$\mathrm{BD}+20^{\circ} 3603$ & 6138 & 3.95 & 3.94 & -2.09 & 1.50 & 0.74 & -52 & -379 & -33 & 384 \\
$\mathrm{BD}+26^{\circ} 2606$ & 6086 & 4.10 & 4.30 & -2.27 & 1.50 & 0.70 & -133 & -100 & 27 & 169 \\
$\mathrm{BD}+28^{\circ} 2137$ & 6135 & 4.25 & 4.22 & -1.93 & 1.50 & 0.70 & 61 & -232 & -135 & 275 \\
$\mathrm{BD}+29^{\circ} 2091$ & 5648 & 4.60 & 4.34 & -1.96 & 1.40 & $<0.70$ & -165 & -338 & 95 & 388 \\
$\mathrm{BD}+34^{\circ} 2476$ & 6247 & 4.10 & 3.97 & -1.94 & 1.50 & 0.77 & -301 & -262 & -133 & 421 \\
$\mathrm{BD}+42^{\circ} 2667$ & 5947 & 4.35 & 4.13 & -1.45 & 1.40 & 0.72 & -118 & -263 & -21 & 289 \\
$\mathrm{BD}+51^{\circ} 1696$ & 5537 & 4.55 & 4.42 & -1.48 & 1.60 & 0.70 & 221 & -270 & 60 & 354 \\
\hline
\end{tabular}

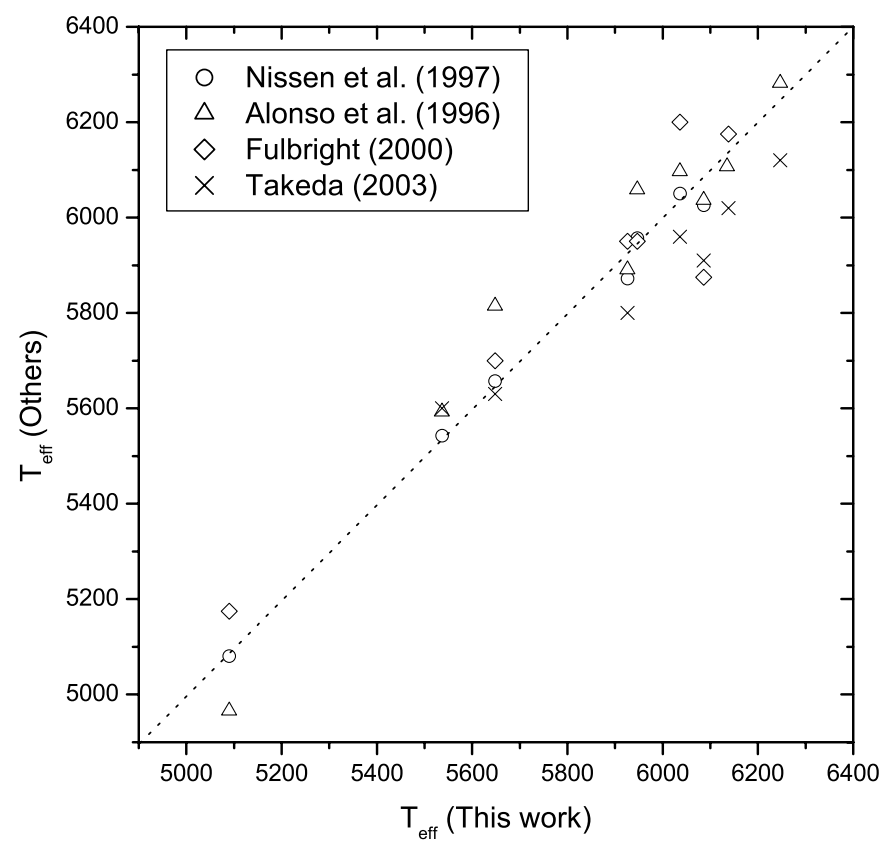

Fig. 2. $T_{\text {eff }}$ comparisons between this work and various literature studies. Circles: Nissen et al. (1997); triangles: Alonso et al. (1996); diamonds: Fulbright (2000); crosses: Takeda (2003).

atmospheric models, the internal errors are derived from the measurement of equivalent widths and atomic data uncertainties. We estimate that the typical uncertainty of the $E W \mathrm{~s}$ is about $3 \mathrm{~m} \AA$ that leads to an internal error of less than 0.1 dex on the derived abundances. Table 3 presents the systematic error for a typical star BD $+42^{\circ} 2667$.

We list the abundance comparison with other authors' results for eight common stars in Table 4. It shows that there is an agreement in iron abundances between this work and other works except for HD 149414, BD $+26^{\circ} 2606$ and $\mathrm{BD}+34^{\circ} 2476$, with differences reaching 0.2 dex. It might be partially caused by the different parameters: lower microturbulent velocity for HD 149414 and lower temperature for BD $+26^{\circ} 2606$ adopted by Fulbright (2000, hereafter F00). For $\mathrm{BD}+34^{\circ} 2476$, our temperature is higher (about $130 \mathrm{~K}$ ) than Stephens \& Boesgaard (2002, hereafter S02) who derived

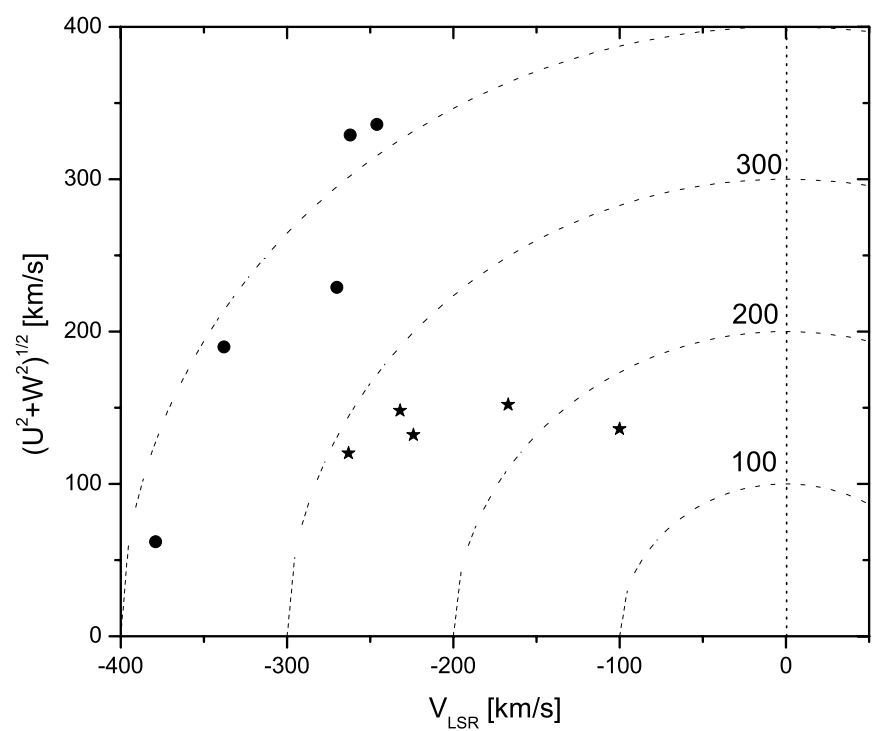

Fig. 3. Toomre diagram: $\left(U^{2}+W^{2}\right)^{1 / 2}$ versus rotational velocity $(V)$. We divide sample stars to two groups: definitively regrograde orbit stars with $V_{\text {tot }}>300 \mathrm{~km} \mathrm{~s}^{-1}$ (filled circles) and remaining stars with $V_{\text {tot }}<300 \mathrm{~km} \mathrm{~s}^{-1}$ (asterisks).

the effective temperature in the abundance versus excitation potential plot.

The $[\mathrm{Mg} / \mathrm{Fe}]$ ratio in the present work is systematically lower than others. We use two weak Mg I lines $4571 \AA$ and $5711 \AA$ and two medium-strong lines $4703 \AA$ and $5528 \AA$, and these four lines give almost consistent $\mathrm{Mg}$ abundance. We believe that these lower abundance of $[\mathrm{Mg} / \mathrm{Fe}]$ is mainly caused by the higher oscillator strengths used in this study. The other elemental abundances $[\mathrm{Si} / \mathrm{Fe}],[\mathrm{Ca} / \mathrm{Fe}],[\mathrm{Ti} / \mathrm{Fe}]$ and $[\mathrm{Cr} / \mathrm{Fe}]$ are generally consistent with the compared data. The discrepancy can be explained by different parameters adopted.

There are large deviations of $\mathrm{Ba}$ abundance between our study and F00 for stars HD 149414 and BD $+20^{\circ} 3603$. In our study, four Ba II lines used for HD 149414 and two Ba II lines used for $\mathrm{BD}+20^{\circ} 3603$ give almost consistent results. For $\mathrm{BD}+20^{\circ} 3603$, the largest deviation between our $[\mathrm{Ba} / \mathrm{Fe}]$ ratio and F00 is unexplained, but Carney et al. (1997) obtained 
Table 3. Abundance errors from atmospheric parameters.

\begin{tabular}{|c|c|c|c|c|c|}
\hline & $\Delta T_{\text {eff }}$ & $\Delta \log g$ & & $\Delta \xi_{t}$ & $\sigma_{\text {tot }}$ \\
\hline & +100 & +0.3 & +0.3 & +0.3 & \\
\hline$\Delta[\mathrm{Fe} / \mathrm{H}]$ & 0.06 & -0.02 & 0.01 & -0.04 & 0.08 \\
\hline$\Delta[\mathrm{O} / \mathrm{Fe}]$ & -0.07 & 0.10 & -0.02 & 0.00 & 0.12 \\
\hline$\Delta[\mathrm{Na} / \mathrm{Fe}]$ & 0.10 & -0.16 & 0.01 & -0.04 & 0.19 \\
\hline$\Delta[\mathrm{Mg} / \mathrm{Fe}]$ & 0.07 & -0.05 & 0.01 & -0.02 & 0.09 \\
\hline$\Delta[\mathrm{Si} / \mathrm{Fe}]$ & 0.04 & 0.02 & 0.01 & 0.00 & 0.05 \\
\hline$\Delta[\mathrm{Ca} / \mathrm{Fe}]$ & 0.05 & -0.03 & 0.01 & -0.03 & 0.07 \\
\hline$\Delta[\mathrm{Sc} / \mathrm{Fe}]$ & 0.04 & 0.11 & 0.02 & -0.02 & 0.12 \\
\hline$\Delta[\mathrm{Ti} / \mathrm{Fe}]$ & 0.07 & 0.00 & 0.01 & -0.02 & 0.07 \\
\hline$\Delta[\mathrm{Cr} / \mathrm{Fe}]$ & 0.06 & -0.04 & 0.04 & -0.02 & 0.08 \\
\hline$\Delta[\mathrm{Mn} / \mathrm{Fe}]$ & 0.06 & 0.00 & 0.00 & -0.01 & 0.06 \\
\hline$\Delta[\mathrm{Ba} / \mathrm{Fe}]$ & 0.05 & 0.06 & 0.03 & -0.07 & 0.11 \\
\hline
\end{tabular}

$[\mathrm{Ba} / \mathrm{Fe}]=-0.18 \pm 0.09$ based on the $\lambda 4554$ line, and $[\mathrm{Ba} / \mathrm{Fe}]=$ $-0.12 \pm 0.11$ based on the weaker $\mathrm{Ba}$ II lines. This result is closer to our $[\mathrm{Ba} / \mathrm{Fe}]$ ratio than F00. For the remaining common stars the agreements of $[\mathrm{Ba} / \mathrm{Fe}]$ ratios with $\mathrm{F} 00$ are reasonable.

\section{Results and discussions}

The elements abundances in metal-poor stars as a function of the early history of the Galaxy will be used in the interpretation of Galactic evolution models and nucleosynthesis theory. Table 5 summarized the derived abundances in this study. Solar photospheric abundances were taken from Grevesse \& Sauval (1998). As shown in Fig. 3, to investigate the abundance pattern for stars on retrograde orbits, we present different symbols in the following figures.

\subsection{Oxygen}

The oxygen abundances of metal-poor stars are very important for our understanding of stellar and Galactic evolution. The relation of $[\mathrm{O} / \mathrm{Fe}]$ vs. $[\mathrm{Fe} / \mathrm{H}]$ has been presented and discussed in many papers using different methods. All authors agree that $[\mathrm{O} / \mathrm{Fe}]$ increases when $[\mathrm{Fe} / \mathrm{H}]$ decreases from 0 to -1 , but the slope of the $[\mathrm{O} / \mathrm{Fe}]$ versus $[\mathrm{Fe} / \mathrm{H}]$ dependence and its behavior for metallicities below -1 are inconsistent.

Abia \& Rebolo (1989) derived oxygen abundance by analysis of the $\mathrm{O}_{\mathrm{I}}$ infrared triplets in 30 unevolved field stars with metallicities in the range $-3.5 \leq[\mathrm{Fe} / \mathrm{H}] \leq-0.2$ and found that $[\mathrm{O} / \mathrm{Fe}]$ reaches $1.0-1.2$ at $[\mathrm{Fe} / \mathrm{H}]=-2$. That result is significantly higher than those in dwarfs using the forbidden line $6300 \AA$ A by Spite \& Spite (1991). Recently, the study by Mishenina et al. (2000) derived oxygen abundance through the O I $7774 \AA$ triplets from the non-LTE determination. The average $[\mathrm{O} / \mathrm{Fe}]$ is $0.61 \pm 0.21 \mathrm{dex}$, and they found a trend of increasing oxygen abundance with decreasing iron abundance in the range $-2.5<[\mathrm{Fe} / \mathrm{H}]<-0.5$. However, Nissen et al. (2002) suggested a more or less flat trend of $[\mathrm{O} / \mathrm{Fe}]$ in the metallicity range of $-2.0<[\mathrm{Fe} / \mathrm{H}]<-1.0$ calculated using the $6300 \AA$ forbidden line based on the new generation of 3D hydrodynamical model atmospheres (Asplund \& García Pèrez 2001).

We have determined oxygen abundances from $\mathrm{O}$ I triplets at 7771,7774 and $7775 \AA$ by considering both LTE and non-LTE effects. The solar abundance of $\mathrm{O}$ is taken from Grevesse \& Sauval (1998): $\log \epsilon(\mathrm{O})=8.83$. We use oscillator strengths of $\log g f=0.333,0.188$ and -0.034 from the NIST database. The non-LTE corrections for $\mathrm{O}$ I triplets are calculated by Eq. (1) of Takeda (2003) using the EW and two coefficients $(a, b)$ for each combination of $\left(T_{\text {eff }}, \log g, \xi_{t}\right)$ which are summarized in Tables $4-6$ of Takeda (2003). The absolute mean value of the correction is less than 0.1 dex with a maximum of 0.11 dex. The final results are given in Table 6 . Our non-LTE $[\mathrm{O} / \mathrm{Fe}]$ ratios are generally lower than the results ginven by Takeda (2003, Table 5) for the seven common stars (see Table 7). This is due to the discrepancy in both iron abundances and $\mathrm{O}$ abundances. Takeda (2003) used lower $[\mathrm{Fe} / \mathrm{H}]$ but obtained large $\log \epsilon(\mathrm{O})$ based on the published equivalentwidth data taken from the literature. We note that, for four common stars, our equivalent widths of $\mathrm{O}$ I triplets are significantly lower than those in Takeda (2003), as observed by Tomkin et al. (1992). This is the main reason for the higher $[\mathrm{O} / \mathrm{Fe}]$ ratios in Takeda (2003). Figure 4 shows the spectra of the triplet for three program stars, and the comparison with Zhang \& Zhao (2003) for the common star HD 108177 observed using the $2.16 \mathrm{~m}$ telescope at the National Astronomical Observatories (Xinglong, China). Note that the $\mathrm{O}$ I triplet lines are detectable in our spectra except for the third line $7775.395 \AA$ which is comparable with the noise level in the comtinuum at this region. For HD 108177, the EWs of triplets from Zhang \& Zhao (2003) are 33.5, 27.6 and $14.8 \mathrm{~m} \AA$ respectively. These values are close to ours. On the other hand, different stellar parameters and different line lists and oscillator strengths for iron also might be a reason for the $[\mathrm{O} / \mathrm{Fe}]$ ratio deviation. Especially, the effective temperature adopted in this study is systematically higher than Takeda (2003) by about $100 \mathrm{~K}$. It will systematically decrease our $[\mathrm{O} / \mathrm{Fe}]$ ratio by about $0.07 \mathrm{dex}$ (see Table 3 ).

Figure 5a shows our non-LTE results with the mean value of $[\mathrm{O} / \mathrm{Fe}]=0.61 \pm 0.05$. It agrees well with Mishenina et al. (2000) but the trend is not consistent. In Fig. 5b, we compare our results with other oxygen abundances derived from different investigations. We can see that our stars (filled triangles) have nearly constant $[\mathrm{O} / \mathrm{Fe}]$ of $\sim 0.6$ dex in our metalicity range $-2.3<[\mathrm{Fe} / \mathrm{H}]<-1.4$. This trend supports the flat trend by Nissen et al. (2002), although the mean [O/Fe] ratio is about 0.3 dex higher than theirs, and the metallicity coverage is not large enough.

\section{2. $\alpha$-elements (Mg, Si, Ca and Ti)}

The bulk of $\alpha$-elements was produced by type II supernovae originating from massive stars (Arnett 1978). The nearly constant ratios between the $\alpha$-elements and $\mathrm{Fe}$ in the metallicity range $-3.2 \leq[\mathrm{Fe} / \mathrm{H}] \leq-1.8$ are observed by 
Table 4. The comparison of stellar parameters and abundances between this work and previous studies for common stars.

\begin{tabular}{|c|c|c|c|c|c|c|c|c|c|c|c|c|c|c|c|c|c|c|}
\hline \multirow[t]{2}{*}{ Star } & \multicolumn{2}{|c|}{ HD108177 } & \multicolumn{2}{|c|}{ HD149414 } & \multicolumn{3}{|c|}{$\mathrm{BD}+02^{\circ} 3375$} & \multicolumn{3}{|c|}{$\mathrm{BD}+20^{\circ} 3603 \mathrm{BD}+26^{\circ}$} & \multicolumn{2}{|c|}{$5^{\circ} 2606 \mathrm{BD}+29$} & \multicolumn{3}{|c|}{$2091 \mathrm{BD}+34^{\circ} 2476$} & \multicolumn{3}{|c|}{$\mathrm{BD}+42^{\circ} 2667$} \\
\hline & TUV & F00 & TW & F00 & $\mathrm{TW}$ & 500 & $\mathrm{~S} 02$ & TW & F00 & TW & F00 & TW & F00 & TW & S02 & TW & F00 & S02 \\
\hline & 6036 & 6200 & 5090 & 5175 & 5926 & 5950 & 5960 & 6138 & 6175 & 6086 & 5875 & 5648 & 5700 & 6247 & 6118 & 5947 & 5950 & 6108 \\
\hline 1089 & 4 & 4.40 & 4 & U & 4.20 & 4.20 & 4. & 3 & 4.00 & 4 & 4 & 4 & 4. & 4 & 5 & $4.3 J$ & $4.2 \mathrm{C}$ & 4. \\
\hline$\xi_{t}$ & 1.50 & 1.35 & 1.50 & 0.30 & 1.50 & 1.00 & 1.52 & 1.50 & 0.90 & 1.50 & 0.40 & 1.40 & 1.30 & 1.50 & 1.45 & 1.40 & 1.20 & 1.43 \\
\hline$[\mathrm{Fe} / \mathrm{H}]$ & -1.69 & -1.65 & -1.51 & -1 & -2.25 & -2.32 & -2.33 & -2.09 & -2.11 & 27 & -2.48 & - & 98 & 4 & .18 & 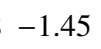 & .48 & -1.40 \\
\hline$/ \mathrm{Fe}]$ & 0.09 & 0.43 & 0.22 & 0.41 & 0.24 & 0.52 & 0.34 & 0.36 & 0.46 & 0.25 & 0.42 & 0.11 & 0.38 & 30.17 & 0.27 & 0.09 & 0.41 & 0.36 \\
\hline 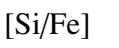 & P & 0.64 & 0.45 & 5 & 0 & - & - & 3 & - & 0 & - & 0.40 & - & 9 & - & 11 & 0.44 & 0 . \\
\hline$[\mathrm{Ca} / \mathrm{Fe}]$ & 0.32 & 0.36 & 0.45 & 0.27 & 0.36 & 0.38 & 0.39 & 0.37 & 0.39 & 0.28 & 0.37 & 0.24 & $\quad 0.42$ & 0.34 & 0.49 & 0.32 & 0.38 & 0.33 \\
\hline $\mathrm{Fe}]$ & 0 & 0.44 & 0 & 9 & 0. & 2 & 0.38 & 0 & 0.48 & 0.63 & 0.52 & 0. & 0.54 & 0.43 & 0.44 & 0.39 & 0.36 & 0.31 \\
\hline$[\mathrm{Cr} / \mathrm{Fe}]$ & -0.02 & -0.02 & 0.02 & 0.06 & 0.05 & 0.00 & 0.13 & 0.05 & -0.05 & -0.05 & -0.12 & -0.06 & -0.09 & 0.07 & 0.05 & -0.03 & -0.04 & -0.04 \\
\hline$[\mathrm{Ba} / \mathrm{Fe}]$ & 0.01 & 0.02 & 0.28 & 0.06 & -0.27 & -0.25 & -0.29 & 0.04 & -0.46 & -0.42 & -0.32 & 0.05 & - & 0.01 & 0.02 & 0.12 & 0.09 & 0.32 \\
\hline
\end{tabular}

TW: This work; F00: Fulbright (2000); S02: Stephens \& Boesgaard (2002).

Table 5. Elemental abundances.

\begin{tabular}{lccccccccccc}
\hline \hline Star & {$[\mathrm{Fe} / \mathrm{H}]$} & {$[\mathrm{O} / \mathrm{Fe}]$} & {$[\mathrm{Na} / \mathrm{Fe}]$} & {$[\mathrm{Mg} / \mathrm{Fe}]$} & {$[\mathrm{Si} / \mathrm{Fe}]$} & {$[\mathrm{Ca} / \mathrm{Fe}]$} & {$[\mathrm{Sc} / \mathrm{Fe}]$} & {$[\mathrm{Ti} / \mathrm{Fe}]$} & {$[\mathrm{Cr} / \mathrm{Fe}]$} & {$[\mathrm{Mn} / \mathrm{Fe}]$} & {$[\mathrm{Ba} / \mathrm{Fe}]$} \\
\hline $\mathrm{HD} 108177$ & -1.69 & 0.65 & -0.29 & 0.09 & 0.46 & 0.32 & 0.07 & 0.47 & -0.02 & -0.35 & 0.01 \\
$\mathrm{HD} 149414$ & -1.51 & 0.69 & -0.45 & 0.22 & 0.45 & 0.45 & 0.15 & 0.36 & 0.02 & -0.23 & 0.28 \\
$\mathrm{BD}+02^{\circ} 3375$ & -2.25 & 0.59 & -0.29 & 0.24 & 0.54 & 0.36 & 0.09 & 0.48 & 0.05 & -0.42 & -0.27 \\
$\mathrm{BD}+20^{\circ} 3603$ & -2.09 & 0.66 & -0.44 & 0.36 & 0.73 & 0.37 & 0.17 & 0.53 & 0.05 & -0.27 & 0.04 \\
$\mathrm{BD}+26^{\circ} 2606$ & -2.27 & 0.68 & -0.49 & 0.25 & 0.40 & 0.28 & 0.23 & 0.63 & -0.05 & -0.27 & -0.42 \\
$\mathrm{BD}+28^{\circ} 2137$ & -1.93 & 0.56 & -0.34 & 0.21 & 0.48 & 0.34 & 0.15 & 0.43 & 0.01 & -0.23 & 0.03 \\
$\mathrm{BD}+29^{\circ} 2091$ & -1.96 & 0.56 & -0.59 & 0.11 & 0.40 & 0.24 & 0.23 & 0.38 & -0.06 & -0.52 & 0.05 \\
$\mathrm{BD}+34^{\circ} 2476$ & -1.94 & 0.60 & -0.43 & 0.17 & 0.59 & 0.34 & 0.12 & 0.43 & 0.07 & -0.41 & 0.01 \\
$\mathrm{BD}+42^{\circ} 2667$ & -1.45 & 0.61 & -0.31 & 0.09 & 0.17 & 0.32 & 0.09 & 0.39 & -0.03 & -0.21 & 0.12 \\
$\mathrm{BD}+51^{\circ} 1696$ & -1.48 & 0.53 & -0.61 & 0.18 & 0.35 & 0.29 & 0.15 & 0.30 & 0.04 & -0.20 & 0.08 \\
\hline
\end{tabular}

Table 6. Equivalent widths and abundances of $\mathrm{O}$.

\begin{tabular}{lccccccccc}
\hline \hline Star & \multicolumn{7}{c}{$E W(\mathrm{~mA})$} & \multicolumn{3}{c}{$[\mathrm{O} / \mathrm{Fe}]$} & $\Delta_{\text {NLTE }}$ & {$[\mathrm{O} / \mathrm{Fe}]$} \\
& $7771 / 7774 / 7775$ & LTE & $7771 /$ & $7774 /$ & 7775 & NLTE \\
\hline HD 108177 & 34.0 & 24.3 & 10.8 & 0.74 & -0.10 & -0.09 & -0.08 & 0.65 \\
HD 149414 & 9.9 & 7.0 & 2.4 & 0.70 & -0.01 & -0.01 & -0.01 & 0.69 \\
$\mathrm{BD}+02^{\circ} 3375$ & 9.6 & 5.4 & 3.8 & 0.66 & -0.08 & -0.07 & -0.07 & 0.59 \\
$\mathrm{BD}+20^{\circ} 3603$ & 19.4 & 15.4 & - & 0.75 & -0.09 & -0.08 & - & 0.66 \\
$\mathrm{BD}+26^{\circ} 2606$ & 11.0 & 7.5 & 6.2 & 0.75 & -0.08 & -0.08 & -0.07 & 0.68 \\
$\mathrm{BD}+28^{\circ} 2137$ & 25.3 & 12.3 & 7.7 & 0.64 & -0.09 & -0.08 & -0.07 & 0.56 \\
$\mathrm{BD}+29^{\circ} 2091$ & 6.3 & - & - & 0.58 & -0.02 & - & - & 0.56 \\
$\mathrm{BD}+34^{\circ} 2476$ & 25.9 & 21.9 & 12.4 & 0.69 & -0.10 & -0.09 & -0.08 & 0.60 \\
$\mathrm{BD}+42^{\circ} 2667$ & 35.5 & 29.0 & 18.7 & 0.70 & -0.11 & -0.10 & -0.09 & 0.61 \\
$\mathrm{BD}+51^{\circ} 1696$ & 14.0 & - & - & 0.55 & -0.02 & - & - & 0.53 \\
\hline
\end{tabular}

Nissen et al. (1994). However, a more precise analysis of $[\alpha / \mathrm{Fe}]$ shows a pronounced scatter in the metal-poor stars that has been interpreted mostly as a consequence of the inhomogeneity of the matter making up the stars rather than a result of poor observational data (Audouze \& Silk 1995). For all four $\alpha$-element ratios, Stephens \& Boesgaard (2002) found high values at low metallicities that decrease to near-solar values at high metallicities for metal-poor halo dwarfs with unusual orbital properties (e.g. stars coming from the outskirts of the halo or stars on retrograde orbits).

Our results for the $\alpha$-elements $(\mathrm{Mg}, \mathrm{Si}, \mathrm{Ca}$ and $\mathrm{Ti})$ are found to be overabundant by $0.19 \pm 0.08,0.46 \pm 0.15,0.33 \pm 0.06$, and $0.44 \pm 0.09$ dex respectively. As seen in Fig. 6, all of the four $\alpha$-elements show a slightly decreasing trend with increasing iron abundance $[\mathrm{Fe} / \mathrm{H}]$ in our metallicity range with different slope, and there are no significantly different pattern between the two groups of halo stars with different kinematics, definitively retrograde stars (filled circles) and remaining stars (asterisks). For silicon, only fewer than five weak $\mathrm{Si}$ I lines are available and all $E W$ values less than $10 \mathrm{~m} \AA$, in the metallicity range of $[\mathrm{Fe} / \mathrm{H}]<-1.9$ dex. Moreover, these Si lines have not provided accurate oscillator strengths. This caused the larger 
Table 7. Oxygen abundance comparison with Takeda (2003).

\begin{tabular}{|c|c|c|c|c|c|c|}
\hline \multirow[t]{2}{*}{ Star } & $T_{\text {eff }}$ & \multicolumn{2}{|c|}{$[\mathrm{Fe} / \mathrm{H}]$} & \multirow{2}{*}{$\frac{\log \epsilon(\mathrm{O})}{\mathrm{TW} \text { T03 }}$} & \multicolumn{2}{|c|}{$[\mathrm{O} / \mathrm{Fe}]_{\mathrm{NLTE}}$} \\
\hline & TW T03 & TW & T03 & & TW & T03 \\
\hline D 108177 & 60365 & -1.69 & -1.87 & 7.79 & 0.65 & 0.78 \\
\hline $\mathrm{BD}+02^{\circ} 3375$ & 59265800 & -2.25 & -2.40 & 7.177 .51 & 0.59 & 0.98 \\
\hline $\mathrm{BD}+20^{\circ} 3603$ & 61386020 & -2.09 & -2.17 & 7.487 .40 & 0.66 & 0.72 \\
\hline $\mathrm{BD}+26^{\circ} 2606^{*}$ & * 60865980 & -2.27 & -2.40 & 7.247 .67 & 0.68 & 1.14 \\
\hline $\mathrm{BD}+29^{\circ} 2091$ & 56485630 & -1.96 & -2.20 & 7.437 .71 & 0.56 & 0.98 \\
\hline $\mathrm{BD}+34^{\circ} 2476$ & 62476120 & -1.94 & -2.19 & 7.497 .52 & 0.60 & 0.78 \\
\hline $\mathrm{BD}+51^{\circ} 1696$ & 55375600 & -1.48 & -1.49 & 7.888 .15 & 0.53 & 0.71 \\
\hline
\end{tabular}

TW: This work; T03: Takeda (2003).

* : For this star the reanalysis data used by Takeda is from Abia \& Rebolo (1989), and remaining stars' data are from Tomkin et al. (1992).
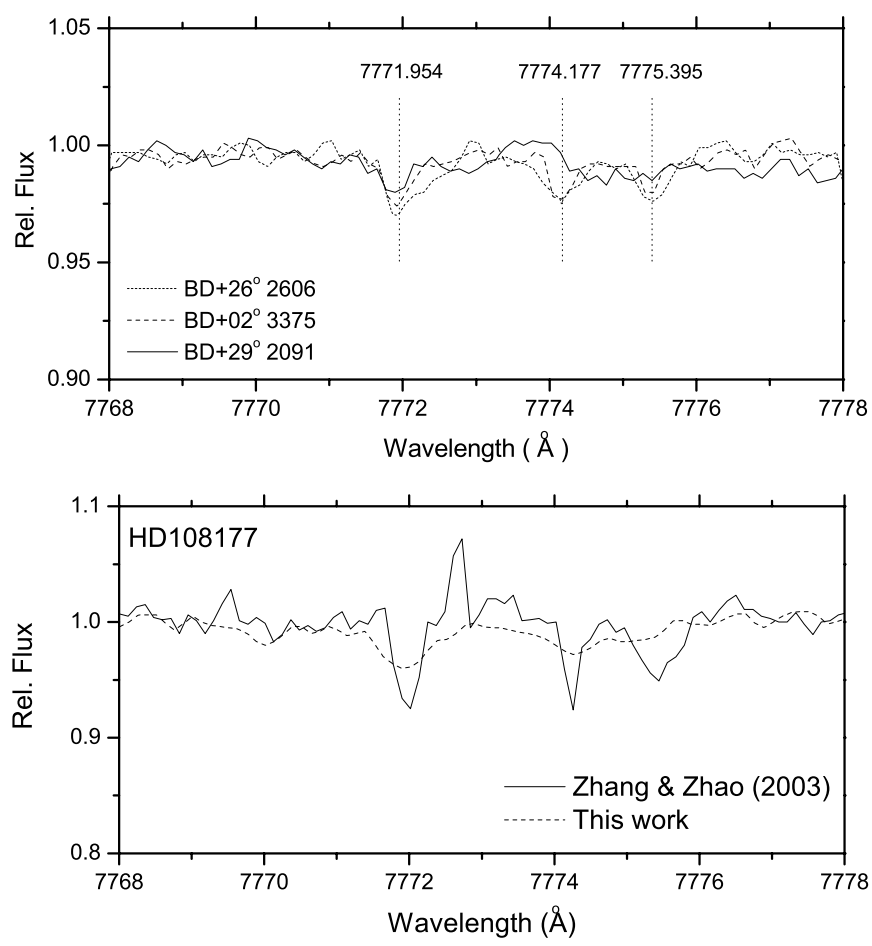

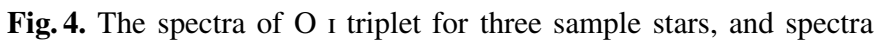
comparison with Zhang \& Zhao (2003) for HD 108177.

scatter of Si abundance than other $\alpha$-elements. $\mathrm{Ca}$ and $\mathrm{Ti}$ are measured with more than 10 neutral lines, and the abundances relative to iron show a consistant trend with smaller scatter.

\subsection{Sodium ( $\mathrm{Na})$}

The odd-Z element Na was thought to be synthesized in massive stars like the $\alpha$-elements, but $\mathrm{Na}$ is primarily manufactured during supernova explosions (Timmes et al. 1995). Therefore, the $[\mathrm{Na} / \mathrm{Mg}]$ ratio declines as a near mirror image of the increase of $[\mathrm{Mg} / \mathrm{Fe}]$ with decreasing $[\mathrm{Fe} / \mathrm{H}]$ in the disk and halo (Wheeler et al. 1989). Stephens \& Boesgaard (2002) also found out that the ratios of $[\mathrm{Na} / \mathrm{Fe}]$ and $[\mathrm{Mg} / \mathrm{Fe}]$ increase together.
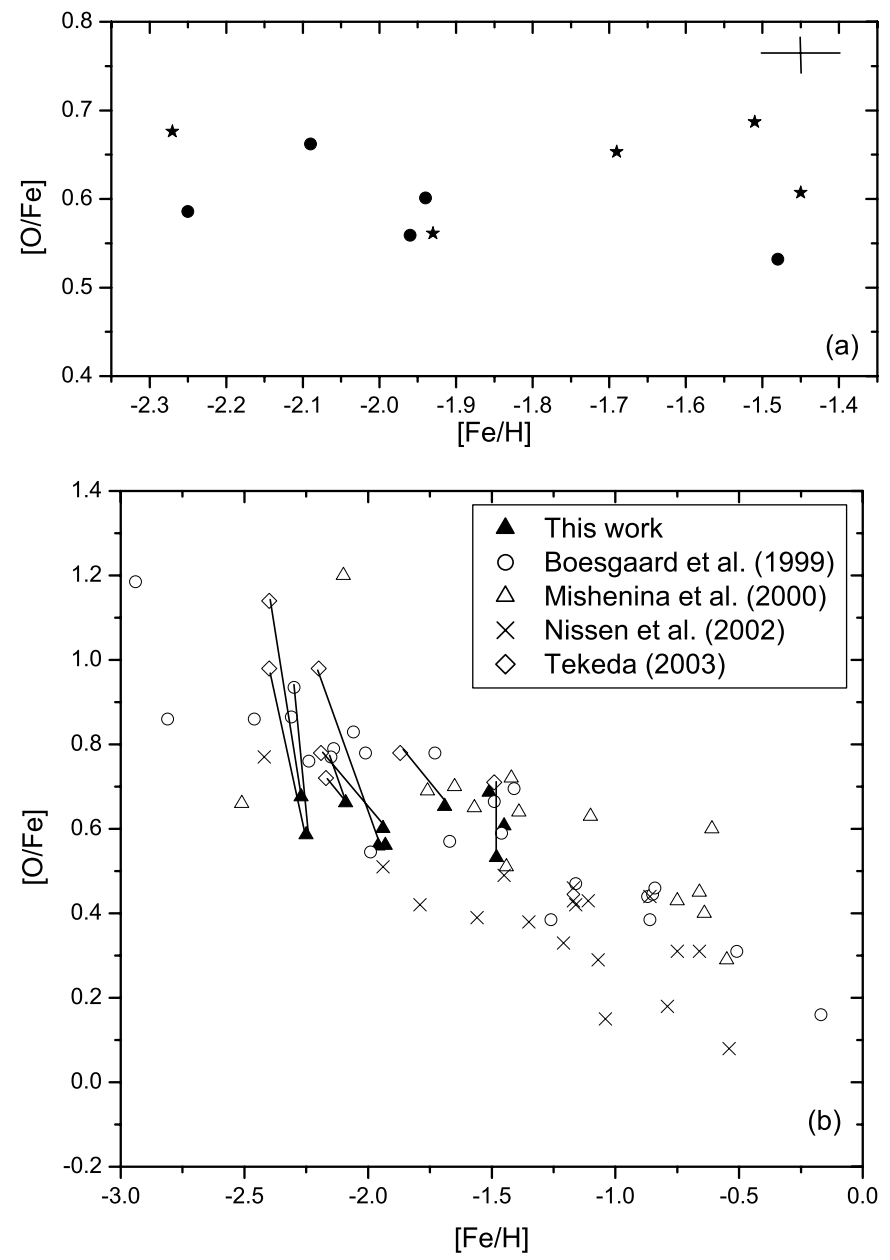

Fig. 5. a): The $[\mathrm{O} / \mathrm{Fe}]$ ratio vs. $[\mathrm{Fe} / \mathrm{H}]$ of our sample stars. b): Comparison with other studies. The open circles present the data from Beosgaard et al. (1999) using $\mathrm{OH}$ lines. The open triangles present $\mathrm{O}_{\mathrm{I}}$ triplets result from Mishenina et al. (2000), and the crosses present the forbidden line [O I] $6300 \AA$ result from Nissen et al. (2002). The diamonds present reanalysed $[\mathrm{O} / \mathrm{Fe}]$ ratio by Takeda (2003) for common stars. Our results are presented by filled triangles. The common stars are connected with solid lines.

Previous analysis of sodium under the assumption of LTE for metal-poor stars gave solar abundance (McWilliam et al. 1995). Recently, Baumüller et al. (1998, hereafter B98) analyzed the full non-LTE line formation of sodium to determine the nonLTE effect of neutral sodium. They derived the solar $[\mathrm{Na} / \mathrm{Fe}]$ ratio by LTE analysis and obtained a lower $[\mathrm{Na} / \mathrm{Fe}]$ when nonLTE line formation is taken into account. For the Na I D lines, the LTE abundances can be significantly different from their non-LTE with differences reaching 0.6 dex in extreme cases.

In this study, we only measured the strong Na I D 5889/95 lines to calculate sodium abundance. Their non-LTE corrections are calculated using profile fitting by the synthesis program SIU (see Shi et al. 2004, for details). The mean nonLTE correction reached -0.40 dex that reduces the nearly solar $[\mathrm{Na} / \mathrm{Fe}]$ ratios based on LTE analysis down to underabundance (mean value $-0.42 \pm 0.12 \mathrm{dex}$ ) from nearly solar values. Table 8 lists the non-LTE correction for $\mathrm{Na}$ I $\mathrm{D}$ lines. Note that the non-LTE corrections increase significantly with effective 


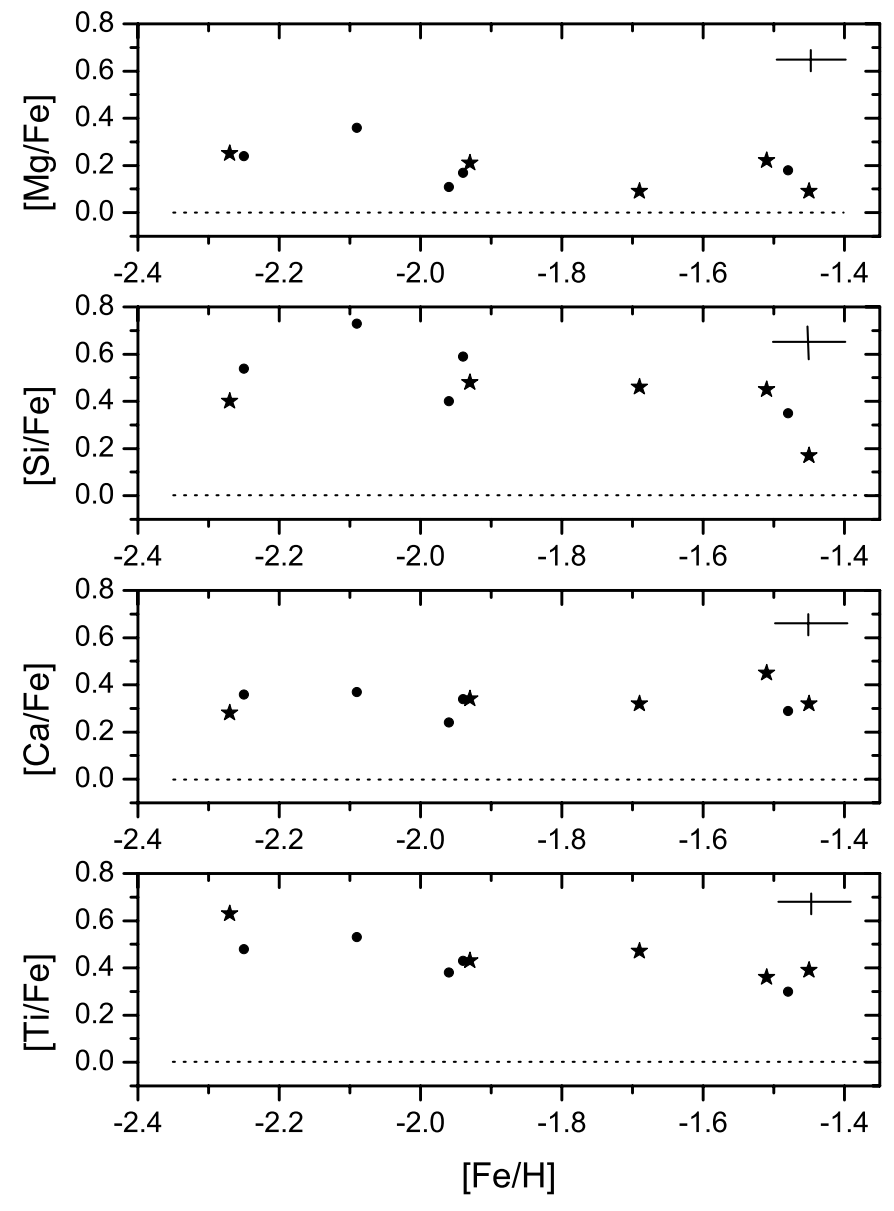

Fig. 6. Abundance patterns for $\alpha$-elements ( $\mathrm{Mg}, \mathrm{Si}, \mathrm{Ca}$ and $\mathrm{Ti})$.

temperature $T_{\text {eff }}$, and decrease with increasing metallicity in the range $[\mathrm{Fe} / \mathrm{H}]>-2.0$, but appear opposite to $[\mathrm{Fe} / \mathrm{H}]<-2.0$, as mentioned in Shi et al. (2004). This explains that the nonLTE effect of $\mathrm{Na}$ I D lines is stronger for hot and metaldeficient stars in the range $[\mathrm{Fe} / \mathrm{H}]>-2.0$. Figure 7 shows our non-LTE result of sodium abundance with B98's sample stars. It shows that there is no obvious trend of the $[\mathrm{Na} / \mathrm{Fe}]$ vs. $[\mathrm{Fe} / \mathrm{H}]$ diagram in the metallicity range of $-2.3<[\mathrm{Fe} / \mathrm{H}]<$ -1.4 . This is not inconsistent with B98's study since we have no stars with $[\mathrm{Fe} / \mathrm{H}]<-2.3$ : in $\mathrm{B} 98$, the tentative decreasing $[\mathrm{Na} / \mathrm{Fe}]$ with $[\mathrm{Fe} / \mathrm{H}]$ appears at $[\mathrm{Fe} / \mathrm{H}]<-2.5$. However, the $[\mathrm{Na} / \mathrm{Fe}]$ values in the present work are statistically lower than that of B98 by order of 0.15 dex for the same metallicity range of $-2.3<[\mathrm{Fe} / \mathrm{H}]<-1.4$. But this result needs further investigation because we notice that the non-LTE corrections between the present study and B98 are somewhat different although both works adopt the same profile fitting method. Observationally the lower $[\mathrm{Na} / \mathrm{Fe}]$ in retrograde stars could be real. It has been suggested by Hanson et al. (1998) that some retrograde giants in the halo have low $[\mathrm{Na} / \mathrm{Fe}] \mathrm{ra}-$ tios $(<-0.35$ dex $)$ which is confirmed in retrograde halo dwarfs by the present study if the comparison of $[\mathrm{Na} / \mathrm{Fe}]$ between the present work and B98 is internally consistent. No obvious difference is found between definitive retrograde stars and the remaining stars based on the present work.
Table 8. Non-LTE corrections and results for Na I D lines.

\begin{tabular}{lccccccc}
\hline \hline Star & \multicolumn{2}{c}{$[\mathrm{Na} / \mathrm{Fe}]_{\mathrm{LTE}}$} & \multicolumn{2}{c}{$[\mathrm{Na} / \mathrm{Fe}]_{\mathrm{NLTE}}$} & $\Delta_{\mathrm{NLTE}}$ & {$[\mathrm{Na} / \mathrm{Fe}]$} \\
& $5889 / 95 \AA$ & $5889 / 95 \AA$ & Mean & NLTE \\
\hline $\mathrm{HD} 108177$ & 0.12 & 0.27 & -0.33 & -0.25 & -0.48 & -0.29 \\
$\mathrm{HD} 149414$ & -0.36 & -0.28 & -0.48 & -0.42 & -0.13 & -0.45 \\
$\mathrm{BD}+02^{\circ} 3375$ & 0.07 & 0.24 & -0.35 & -0.23 & -0.45 & -0.29 \\
$\mathrm{BD}+20^{\circ} 3603$ & 0.12 & 0.11 & -0.48 & -0.40 & -0.56 & -0.44 \\
$\mathrm{BD}+26^{\circ} 2606$ & -0.10 & -0.11 & -0.53 & -0.45 & -0.39 & -0.49 \\
$\mathrm{BD}+28^{\circ} 2137$ & 0.20 & 0.14 & -0.35 & -0.33 & -0.51 & -0.34 \\
$\mathrm{BD}+29^{\circ} 2091$ & -0.32 & -0.18 & -0.62 & -0.56 & -0.34 & -0.59 \\
$\mathrm{BD}+34^{\circ} 2476$ & 0.22 & 0.04 & -0.42 & -0.45 & -0.57 & -0.43 \\
$\mathrm{BD}+42^{\circ} 2667$ & 0.05 & 0.01 & -0.29 & -0.33 & -0.34 & -0.31 \\
$\mathrm{BD}+51^{\circ} 1696$ & -0.43 & -0.31 & -0.65 & -0.57 & -0.24 & -0.61 \\
\hline
\end{tabular}

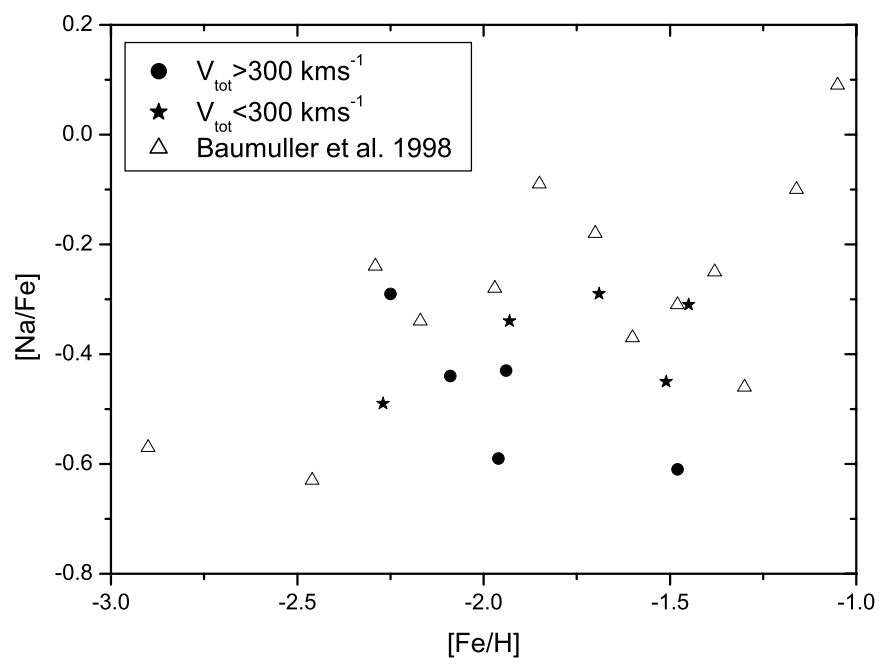

Fig. 7. NLTE abundances of Sodium: filled symbols: this study, open triangles: Baumüller et al. (1998).

\subsection{Scandium (Sc)}

As an element between $\alpha$-elements and iron-peak elements, scandium abundance provides some special constraints on nucleosynthesis theory. However, the observational situation for scandium is not satisfactory at present. Additionally, the significance of the hyperfine structure (HFS) of Sc lines on the abundance pattern of Sc is not well established. Zhao \& Magain (1990) derived the overabundance of Sc relative to Fe without consideration of HFS in metal-poor dwarfs. Gratton \& Sneden (1991) considered HFS for Sc lines using synthetic spectrum computations and found nearly solar $[\mathrm{Sc} / \mathrm{Fe}]$ ratios in metalpoor dwarfs and giants. A more detailed HFS effect for Sc lines is discussed by Nissen et al. (2000). They found that the HFS has a small influence on weak Sc II lines. Their [Sc/Fe] declines from an overabundance $(\sim 0.2)$ at $[\mathrm{Fe} / \mathrm{H}]=-1.4$ to zero at solar metallicity, except for the group of low- $\alpha$ halo stars, which tend to have low values of $[\mathrm{Sc} / \mathrm{Fe}]$.

Fewer than five Sc II lines are measured in this study including $\lambda \lambda$ 4400.390, 4670.413, 5239.823, 5526.821 


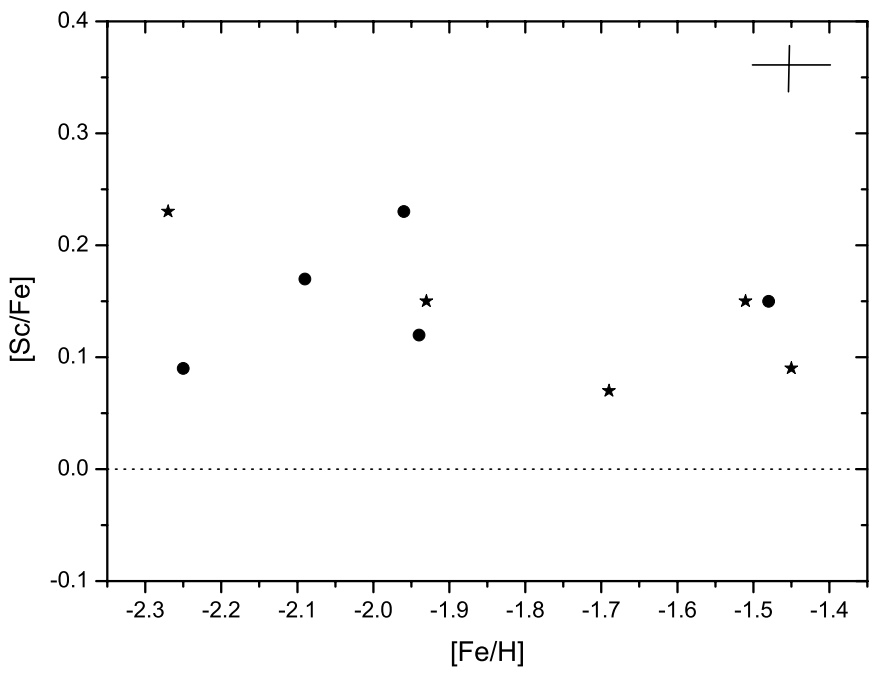

Fig. 8. Scandium abundance versus metallicity.

and $5657.880 \AA$ A. Figure 8 shows Sc abundance results with respect to metallicity without considering the HFS effect. Our results also show that the $\alpha$-elements keep the overabundance and tend to decrease with the increasing metallicity in our range $-2.3<[\mathrm{Fe} / \mathrm{H}]<-1.4$. This agrees with the result of Zhao \& Magain (1990) and supports the investigation of Nissen et al. (2000) into the overabundances of metal-rich halo stars at $[\mathrm{Fe} / \mathrm{H}]=-1.4$. With different HFS data, Prochaska $\&$ McWilliam (2000) re-determined Sc abundances for stars in Nissen et al. (2000) but the enhanced $[\mathrm{Sc} / \mathrm{Fe}]$ is the same. The present result on $[\mathrm{Sc} / \mathrm{Fe}]$ extends the Nissen et al. (2000) work to lower metallicity until $[\mathrm{Fe} / \mathrm{H}]=-2.3$ and confirms a similar nucleosynthesis origin as the $\alpha$-elements.

\subsection{Iron-peak elements ( $\mathrm{Cr}$ and $\mathrm{Mn})$}

The Fe-group elements are mainly produced by explosion of Type I supernova. Previous studies of chromium in stars with any metallicity have yielded the same result: $[\mathrm{Cr} / \mathrm{Fe}] \sim 0.0$ (Wheeler et al. 1989). Zhao \& Magain (1990) obtained nearly solar $\mathrm{Cr}$ abundance $[\mathrm{Cr} / \mathrm{Fe} /]=+0.01 \pm 0.08$ using six weak neutral lines for 20 metal-poor dwarfs. Gratton \& Sneden (1991) obtained a slight underabundance of $[\mathrm{Cr} / \mathrm{Fe}]=-0.09 \pm 0.10$ from $\mathrm{Cr}$ I lines and $[\mathrm{Cr} / \mathrm{Fe}]=-0.04 \pm 0.05$ from $\mathrm{Cr}$ II lines for the metal-poor dwarfs and giants. For the different abundances of $\mathrm{Cr}$ I and $\mathrm{Cr}$ II lines they suggested that some overionization of $\mathrm{Cr}$ occurs in these stars. Our result from $\mathrm{Cr}$ I lines also give nearly solar values with mean $[\mathrm{Cr} / \mathrm{Fe}]=+0.02 \pm 0.05$, but the $\mathrm{Cr}$ II lines give overabundance results that are higher than the $\mathrm{Cr}$ I result by 0.15 dex in extreme cases. We propose that the difference between abundances provided by neutral and singly ionized $\mathrm{Cr}$ lines may be caused by the distribution of the neutral and ionized $\mathrm{Cr}$ in different layers of the atmosphere, with different temperatures and gravities.

Some of the early contributions to stellar abundance studies suggested that the odd- $Z$ elements of the Fe group (at least $\mathrm{V}$ and $\mathrm{Mn}$ ) are deficient in metal-poor stars (Wheeler et al. 1989). It is confirmed by Gratton (1989); he derived a

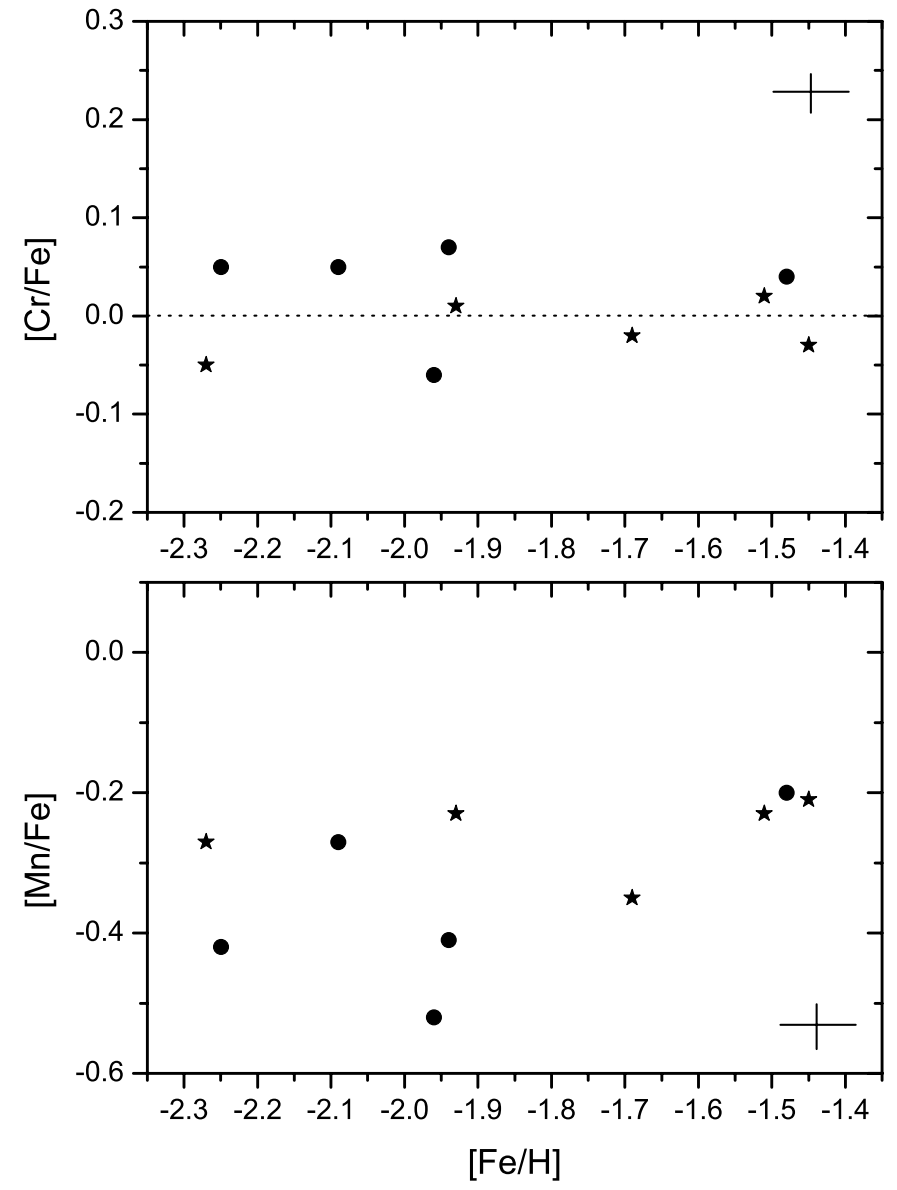

Fig. 9. Iron peak elements ratios as a function of metallicity.

constant $[\mathrm{Mn} / \mathrm{Fe}]$ ratio for $[\mathrm{Fe} / \mathrm{H}]<-1$ with a mean value of $[\mathrm{Mn} / \mathrm{Fe}]=-0.34 \pm 0.06$. Recently, Nissen et al. (2000) published a comprehensive study of [Mn/Fe] ratios for 129 dwarfs in the range $-1.4<[\mathrm{Fe} / \mathrm{H}]<+0.1$ and found the same significant underabundance of $[\mathrm{Mn} / \mathrm{Fe}] \sim-0.5 \mathrm{dex}$ at $[\mathrm{Fe} / \mathrm{H}] \sim-1.4$. In this study, we also obtained an underabundant of [Mn/Fe] ratio with the mean value $[\mathrm{Mn} / \mathrm{Fe}]=-0.31 \pm 0.11$ (see Fig. 9). The HFS effect was not considered in our study. For weak lines the HFS treatment is not so critical because the lines are unsaturated (Prochaska \& McWilliam 2000).

\subsection{Neutron-capture element $\mathrm{Ba}$}

The elements heavier than iron are produced by neutron capture by two main mechanisms according to the strength of the neutron flux. Zhao \& Magain (1995) have reduced barium abundances from two Ba II lines $5853 \AA$ and $6496 \AA$ obtained that $[\mathrm{Ba} / \mathrm{Fe}]$ values are 0.25 dex higher than solar for $[\mathrm{Fe} / \mathrm{H}]>-1.8$, then goes down with decreasing metallicity for $[\mathrm{Fe} / \mathrm{H}]<-1.8$. Mashonkina et al. (1999) analyzed non-LTE effects of $\mathrm{Ba}$ and point out that $[\mathrm{Ba} / \mathrm{Fe}]$ abundance ratios are approximately solar above $[\mathrm{Fe} / \mathrm{H}] \sim-2.2$ where they decrease rapidly by $0.5-0.6 \mathrm{dex}$, and suggest s-process dominated $\mathrm{Ba}$ production, at least for the metal-poor stars with $[\mathrm{Fe} / \mathrm{H}]>-2.2$. We measured four $\mathrm{Ba}$ II lines including 


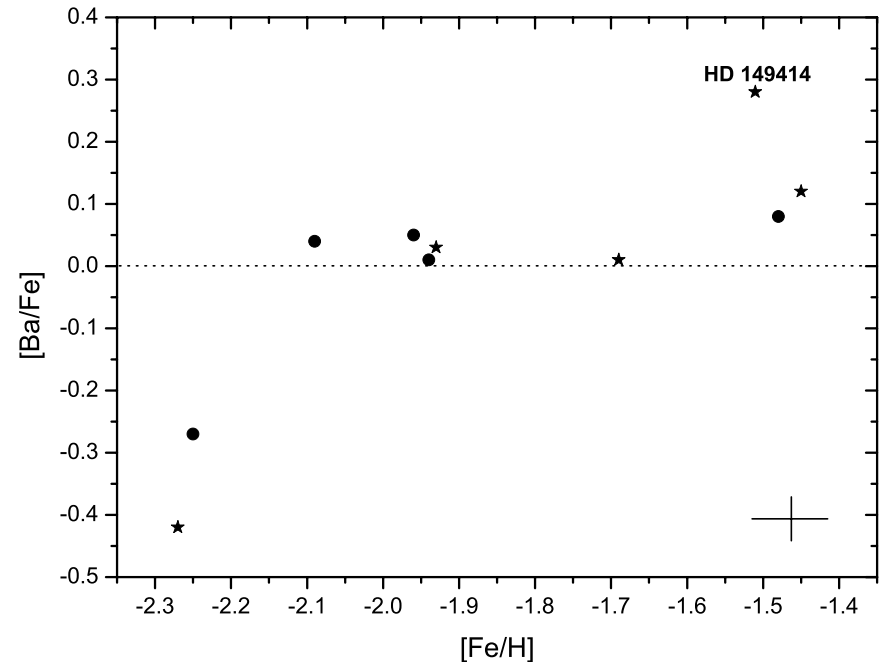

Fig. 10. $[\mathrm{Ba} / \mathrm{Fe}]$ vs. $[\mathrm{Fe} / \mathrm{H}]$.

$\lambda \lambda 4554,5853,6141$ and $6496 \AA$ for our sample stars. For $-2.1<[\mathrm{Fe} / \mathrm{H}]<-1.4,[\mathrm{Ba} / \mathrm{Fe}]$ abundance ratios remain constant within $0.05 \mathrm{dex}$, and decrease rapidly from $[\mathrm{Fe} / \mathrm{H}]<$ -2.1 to $-0.4 \mathrm{dex}$ in $[\mathrm{Fe} / \mathrm{H}] \sim-2.3$ (the Fig. 10). Note that HD 149414, which is a single-lined binary, has significantly a higher $[\mathrm{Ba} / \mathrm{Fe}]$ ratio than nearby stars. Probably it can be explained as a result of mass transfer from its secondary.

\section{Conclusions}

We obtained the chemical composition of 10 halo stars for the 10 elements including $\alpha$-elements $(\mathrm{O}, \mathrm{Mg}, \mathrm{Si}, \mathrm{Ca}$ and $\mathrm{Ti})$, $\mathrm{Na}, \mathrm{Sc}$, iron peak elements ( $\mathrm{Cr}$ and $\mathrm{Mn}$ ) and the neutron capture element $\mathrm{Ba}$. The overabundances of oxygen and other $\alpha$-elements in halo stars are explained by the interaction between the timescale of halo collapse and star formation with the supernova of different original masses. The kinematics indicate that most of the sample stars are on retrograde orbits. The main conclusions are:

(1) In connection with the recent discrepancy (flat or linear increase) in $[\mathrm{O} / \mathrm{Fe}]$ vs. $[\mathrm{Fe} / \mathrm{H}]$ trend, we find that oxygen abundances from $\mathrm{O}_{\mathrm{I}}$ infrared triplets at $\lambda 7774 \AA$ with non-LTE corrections suggest a flat $[\mathrm{O} / \mathrm{Fe}]$ with a metallicity for halo stars of $-2.3<[\mathrm{Fe} / \mathrm{H}]<-1.4$.

(2) We performed non-LTE calculations for $\mathrm{Na}$ I $\mathrm{D}$ lines and indicated the underabundant $[\mathrm{Na} / \mathrm{Fe}]$ ratios. Comparison of the $[\mathrm{Na} / \mathrm{Fe}]$ ratios with $\mathrm{B} 98$ shows a lower $[\mathrm{Na} / \mathrm{Fe}]$ value for retrograde stars than those of normal halo stars which is found by Hanson et al. (1998) for retrograde giants.

(3) There is no special abundance pattern in $\alpha$ elements for retrograde stars. The behaviour of $[\mathrm{Sc} / \mathrm{Fe}]$ versus $[\mathrm{Fe} / \mathrm{H}]$ follows the $\alpha$-elements with the enhanced value of $[\mathrm{Sc} / \mathrm{Fe}]=$ $0.15 \pm 0.06$, and we attribute it as an $\alpha$-element.

(4) The recent studies on $\mathrm{Mn}$ abundances are quite limited. We extend the recent investigation of the $[\mathrm{Mn} / \mathrm{Fe}]$ vs. $[\mathrm{Fe} / \mathrm{H}]$ trend in Nissen et al. (2000) to lower metallicity until $[\mathrm{Fe} / \mathrm{H}] \sim-2.3$ dex.
(5) There is no obvious difference between five stars definitively classified as retrograde stars and the remaining stars in our sample. But three retrograde stars do show lower $[\mathrm{Mn} / \mathrm{Fe}]$ than the other stars at $-2.3<[\mathrm{Fe} / \mathrm{H}]<-1.8$. Interestingly, the lower $[\mathrm{Mn} / \mathrm{Fe}]$ has been suggested as an important property of the thick disk stars (Nissen 2003). It will be important to investigate the connection between the formation of the thick disk and these retrograde stars in future work.

Acknowledgements. We thank the referee Dr. Wako Aoki for useful suggestions. This work is supported by NKBRSF G1999075406, the National Natural Science Foundation of China under grant No. 10203002 and the Russian Foundation for Basic Research 01-02-16093.

\section{References}

Abia, C., \& Rebolo, R. 1989, ApJ, 347, 186

Allende Prieto, C., Lambert, D. L., \& Asplund, M. 2001, ApJ, 556, 63 Alonso, A., Arribas, S., \& Martínez-Roger, C. 1996, A\&A, 117, 227

Alonso, A., Arribas, S., \& Martínez-Roger, C. 1996, A\&A, 313, 873 Arnett, W. D. 1978, ApJ, 219, 1008

Asplund, M., \& García Pérez, A. E. 2001, A\&A, 372, 601

Audouze, J., \& Slik, J. 1995, ApJ, 451, 49

Baumüller, D., Butler, K., \& Gehren, T. 1998, A\&A, 338, 637

Blackwell, D. E., Petford, A. D., Arribas, S., Haddock, D. J., \& Selby, M. J. 1990, A\&A, 232, 396

Boesgaard, A. M., King, J. R., Deliyannis, C. P., \& Vogt, S. S. 1999, AJ, 117, 492

Carney, B. W., Wright, J. S., Sneden, C., et al. 1997, AJ, 114, 363

Chen, Y. Q., Nissen, P. E., Zhao, G., Zhang, H. W., \& Benoni, T. 2000, A\&AS, 141, 491

Fuhrmann, K. 2002, New Astron., 7, 161

Fulbright, J. P. 2000, AJ, 120, 1841

Gilmore, G., \& Wyse, R. F. G. 1998, AJ, 116, 748

Gratton, R. G. 1989, A\&A, 208, 171

Gratton, R. G., \& Sneden, C. 1991, A\&A, 241, 501

Gratton, R. G., Clementini, G., \& Sneden, C. 1997, hipp. Conf., 339

Gratton, R. G., Carretta, E., Desidera, S., et al. 2003, A\&A, 406, 131

Grevesse, N., \& Sauval, A. J. 1998, SSRv, 85, 161

Hanson, R. B., Sneden, C., Kraft, R. P., \& Fulbright, J. 1998, AJ, 116, 1286

Johnson, D. R. H., \& Soderblom, D. R. 1987, AJ, 93, 864

Kurucz, R. L. 1993, CD-ROM 13, ATLAS9 Stellar Atmosphere Programs and $2 \mathrm{~km} \mathrm{~s}^{-1}$ Grid (Cambridge: SAO)

Mashonkina, L., Gehren, T., \& Bikmaev, I. 1999, A\&A, 433, 519

McWilliam, A., Preston, G., Sneden, C., \& Searle, L. 1995, AJ, 109, 2757

Mishenina, T. V., Korotin, S. A., Klochkova, V. G., \& Panchuk, V. E. 2000, A\&A, 353, 978

Nissen, P. E., \& Schuster, W. J. 1991, A\&A, 251, 457

Nissen, P. E., Gustafsson, B., Edvardsson, B., \& Gilmore, G. 1994, A\&A, 285, 440

Nissen, P. E., Høg, E., \& Schuster, W. J. 1997, ESASP, 402, 225

Nissen, P. E., \& Schuster, W. J. 1997, A\&A, 326, 761

Nissen, P. E., Chen, Y. Q., Schuster, W. J., \& Zhao, G. 2000, A\&A, 353, 722

Nissen, P. E., Primas, F., Asplund, M., \& Lambert, D. L. 2002, A\&A, 390,235 
Nissen, P. E. 2003, Carnegie Observatories Astrophysics Series, Vol. 4: Origin and Evolution of the Elements

Nomoto, K., Thielemann, F. W., \& Kiochi, Y. 1984, ApJ, 286, 644

Norris, J. E., Ryan, S. G., \& Beers, T. C. 2001, ApJ, 561, 1034

Peterson, R. C., \& Carney, B. W. 1979, ApJ, 231, 762

Prochaska, J. X., \& McWilliam, A. 2000, ApJ, 537, 57

Ryan, S. G., Norris, J. E., \& Beers, T. C. 1996, ApJ, 471, 254

Shetrone, M. D., Venn, K. A., Tolstoy, E., et al. 2003, AJ, 125, 684

Schuster, W. J., \& Nissen, P. E. 1989, A\&A, 221, 65

Shi, J. R., Gehren, T., \& Zhao, G. 2004, A\&A, 423, 683
Spite, M., \& Spite, F. 1991, A\&A, 252, 689

Stephens, A., \& Boesgaard, A. M. 2002, AJ, 123, 1647

Takeda, Y. 2003, A\&A, 402, 343

Timmes, F. X., Woosley, S. E., \& Weaver, T. A. 1995, ApJS, 98, 617

Tomkin, J., Lemke, M., Lambert, D. L., \& Sneden, C. 1992, AJ, 104, 1568

Wheeler, J. C., Sneden, C., \& Truran, J. W. 1989, ARA\&A, 27, 279

Zhang, H. W., \& Zhao, G. 2003, IAUS, 219, 11

Zhao, G., \& Magain, P. 1990, A\&A, 238, 242

Zhao, G., \& Magain, P. 1995, in ASP Conf. Ser., 81, 574 